\title{
The Effect of Limiting the Scan Range of Computed Tomography Pulmonary Angiography (to Reduce Radiation Exposure) on the Detection of Pulmonary Embolism: A Systematic Review
}

\author{
Amayar Zaw ${ }^{1,2}$, Rebecca Nguyen ${ }^{1,2}$, Leon Lam $^{3}$, Anthony Kaplan ${ }^{2,3}$ and Claudia C. Dobler $1,2,4,5, * \mathbb{C}$ \\ 1 Department of Respiratory and Sleep Medicine, Liverpool Hospital, Sydney 2107, Australia; \\ amayarzaw24@gmail.com (A.Z.); rebecca.han.nguyen@gmail.com (R.N.) \\ 2 South Western Sydney Clinical School, University of New South Wales, Sydney 2107, Australia; \\ anthony.kaplan@health.nsw.gov.au \\ 3 Department of Radiology, Liverpool Hospital, Sydney 2107, Australia; leon.lam@health.nsw.gov.au \\ 4 The George Institute for Global Health, Sydney 2042, Australia \\ 5 Woolcock Institute of Medical Research, The University of Sydney, Sydney 2037, Australia \\ * Correspondence: c.dobler@unsw.edu.au; Tel.: +61-2-8738-3000
}

check for updates

Citation: Zaw, A.; Nguyen, R.; Lam, L.; Kaplan, A.; Dobler, C.C. The Effect of Limiting the Scan Range of Computed Tomography Pulmonary Angiography (to Reduce Radiation Exposure) on the Detection of Pulmonary Embolism: A Systematic Review. Diagnostics 2021, 11, 2179. https://doi.org/10.3390/

diagnostics11122179

Academic Editor: Philippe A. Grenier

Received: 25 October 2021

Accepted: 22 November 2021

Published: 24 November 2021

Publisher's Note: MDPI stays neutral with regard to jurisdictional claims in published maps and institutional affiliations.

Copyright: (c) 2021 by the authors Licensee MDPI, Basel, Switzerland. This article is an open access article distributed under the terms and conditions of the Creative Commons Attribution (CC BY) license (https:// creativecommons.org/licenses/by/ $4.0 /)$.

\begin{abstract}
Background: Computed tomography pulmonary angiography (CTPA) is the standard imaging test for the evaluation of acute pulmonary embolism (PE), but it is associated with patients exposure to radiation. Studies have suggested that radiation exposure can be reduced without compromising PE detection by limiting the scan range (the z-axis, going from up to down); (2) Methods: A literature search was conducted in MEDLINE and EMBASE on 17 July 2021. Studies were included if they enrolled patients who had undergone a CTPA and described the yield of PE diagnoses, number of missed filling defects and/or other diagnoses using a reduced $z$-axis in comparison to a full-length scan. To assess risk of bias, we modified an existing risk of bias tools for observational studies, the Newcastle-Ottawa Scale. Results were synthesized in a narrative review. Primary outcomes were the number of missed PE diagnoses (based on at least one filling defect) and filling defects; the secondary outcome was the number of other missed findings; (3) Results: Eleven cohort studies and one case-control study were included reporting on a total of 3955 scans including 1025 scans with a diagnosis of PE. Six different reduced scan ranges were assessed; the most studied was from the top of the aortic arch to below the heart, in which no PEs were missed (seven studies). One sub-segmental PE was missed when the scan coverage was $10 \mathrm{~cm}$ starting from the bottom of the aortic arch and $14.7 \mathrm{~cm}$ starting from the top of the arch. Five studies that reported on other findings all found that other diagnoses were missed with a reduced $z$-axis. Most of the included studies had a high risk of bias; (4) Conclusions: CTPA scan coverage reduction from the top of aortic arch to below the heart reduced radiation exposure without affecting PE diagnoses, but studies were generally at high risk of bias.
\end{abstract}

Keywords: pulmonary embolism; diagnostic imaging; computed tomography pulmonary angiography radiation; cohort studies; systematic review

\section{Introduction}

Pulmonary embolism (PE) is a common and treatable disease that, if missed, can be associated with high morbidity and mortality, making efficient and accurate diagnosis important to ensure proper patient care [1]. Definitive diagnosis is achieved with the current diagnostic gold standard-computed tomography pulmonary angiography (CTPA) [2]. Relative to other imaging modalities, CTPAs are fast and minimally invasive, highly specific for PE and widely accessible. They are a frequently ordered test to diagnose or exclude PE. In countries such as the United States of America, millions of CTPAs are performed every 
year [3], but less than $10 \%$ of scans performed return positive results [4]. The relatively low yield of the diagnostic test highlights the importance of clinical risk scores to help identify patients with high pre-test probability for $\mathrm{PE}$, but also the importance of weighing up risks and benefits of the imaging test. The most significant risk is the exposure to ionizing radiation. This is of particular concern in young and/or pregnant women, because of a potential long-term increased risk of breast cancer and possible harm to the fetus [5]. Additionally, there is a cumulative risk from repeat scans, as one-third of patients who undergo a CTPA for the first time will undergo a second CTPA within the subsequent five years [6]. Widespread usage of CTPA is also associated with an increased rate of detection of non-PE findings. While identification of other pathologies can be beneficial, for example when identifying alternative etiologies of a patient's symptoms, discovery of incidental findings can also lead to potentially unnecessary further diagnostic investigations and treatments [7].

The widespread use of CTPA warrants careful consideration of strategies to reduce radiation exposure-related risks to patients. Reducing the CTPA $z$-axis (the axis going from up to down) reduces radiation exposure without impairing image quality. As PEs are most commonly found in the lobar and segmental pulmonary arteries in the central portions of the scan [8], there is potential to reduce the boundaries of a scan, such as excluding the lung apices and sub-diaphragmatic regions, while maintaining diagnostic accuracy. However, this would also exclude peripheral areas of the lung containing sub-segmental arteries. As the benefit of treating isolated sub-segmental PEs are unclear [9], it might be reasonable to exclude these regions in order to focus on capturing clinically significant PE that do require treatment.

This is the first systematic review of the effect of a limited CTPA scan coverage on the diagnostic yield for PE and other pathological findings. The goal is to inform clinical practice regarding the utility of reduced CTPA scan coverage in patients in whom reduced radiation exposure is an important consideration.

\section{Materials and Methods}

This protocol adheres to the Preferred Reporting Items for Systematic reviews and Meta-Analyses (PRISMA) guidelines [10].

\subsection{Study Selection}

Studies were included if they enrolled patients who had undergone a CTPA and described the yield of PE diagnoses, number of missed filling defects and/or other diagnoses using a reduced CTPA $z$-axis in comparison to a full-length CTPA scan. We included all publication types, including full text studies, conference abstracts or correspondence with original data in any language. Studies were excluded if they were case reports with fewer than five cases, systematic reviews or narrative reviews.

\subsection{Literature Search}

A systematic search of MEDLINE and EMBASE was performed on 17 July 2021. The search strategy can be found in Table S1. After the removal of duplicates, two reviewers (A.Z. and R.N.) independently assessed the titles and abstracts of all the studies identified by the search against the study selection criteria. For all studies included by either reviewer, the full-text versions were then screened for a final decision about eligibility. The reference lists of the full texts and any identified reviews were examined to find additional relevant studies not identified by the search strategy. In the case of unresolved discrepancies, a third reviewer (C.C.D.) resolved any disagreements.

\subsection{Data Extraction}

We created a data extraction sheet, which was pilot-tested with three studies. A number of modifications were subsequently made to the extraction sheet to ensure all relevant information was captured. Two reviewers (A.Z. and R.N.) extracted all data 
independently. Results were compared and any discrepancies were discussed and resolved, if possible. Any unresolved discrepancies were resolved by a third reviewer (C.C.D.).

\subsection{Quality Assessment}

To assess risk of bias, we modified an existing risk of bias tools for observational studies, the Newcastle-Ottawa Scale, to allow for the fact that the study "subjects" were CTPA scans rather than people (see Table S2). The maximum score per study (representing the lowest risk of bias) was eight points across two domains. First, we assessed the sample size and the proportion of excluded CTPA scans due to image quality (e.g., sub-optimal artery opacification and breathing artefacts). This evaluated the generalizability of the findings to all CTPA scans ordered in clinical practice and also appraised the robustness of the study results. Secondly, we assessed the quality of the process undertaken to analyze the CTPAs - the number of reviewers, their experience and the process of blinding, if any.

\subsection{Outcome Measures}

The primary outcomes measured were the number of PE diagnoses (based on at least one filling defect) and the number of filling defects missed with a reduced CTPA $z$-axis compared to a full-length CTPA. The secondary outcome measured was the number of other non-PE diagnoses missed with the same reduced scan window compared to a full-length CTPA.

\section{Results}

The search strategy identified 563 unique citations, of which 23 studies were included in the full-text screening (Figure 1). Thirteen studies met the inclusion criteria-12 were directly identified by the search algorithm and an additional study was identified via the reference list of one of the included studies. An overview of the studies excluded after full-text screening can be found in Table S4. All included studies were observational studies. Eight studies were published as peer-reviewed articles and five as conference abstracts.

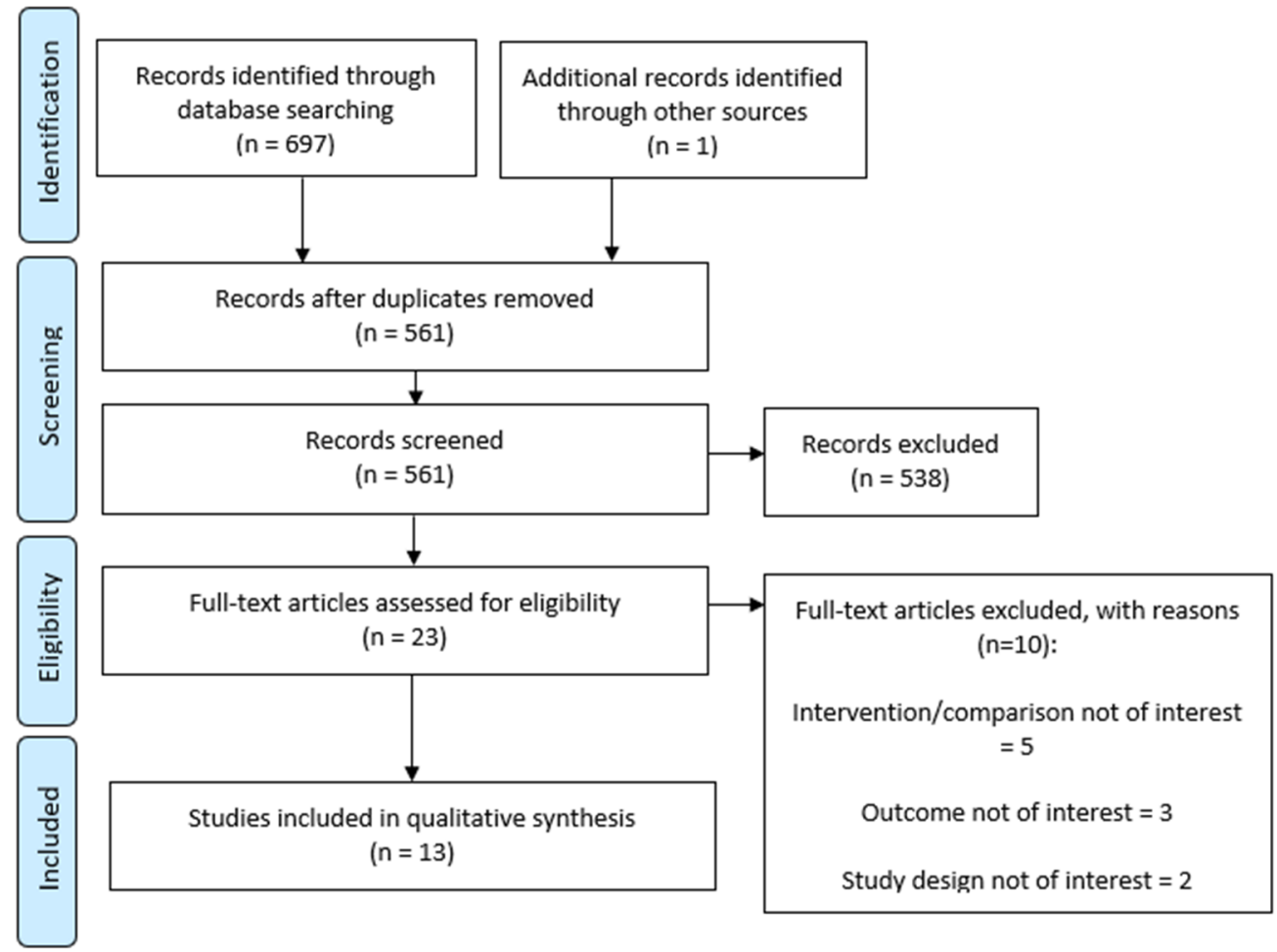

Figure 1. PRISMA flow diagram. 
The characteristics of the included studies are summarized in Table 1, and the CTPA technical parameters and boundaries for the reduced scan window used by each study are described in Table 2. The studies were conducted in the United States of America [11-15] $(n=5)$, Japan [16] $(n=1)$, England [17] $(n=1)$, Belgium [18] $(n=1)$, Australia [19] $(n=1)$ and the Netherlands [20] $(\mathrm{n}=1)$, and the country location of three studies was not specified [21-23]. Eleven studies were conducted in a single hospital (including one in a pediatric center [21]). Of these, two were performed across emergency department (ED), in-patient and out-patient settings [12,15]; one was performed in both ED and in-patient settings [18]; two were performed in an ED setting only [11,14]; and six studies did not specify the patient setting $[13,16,17,19,22]$. Two studies did not specify the type of institution or setting $[20,23]$.

Table 1. Study characteristics of included studies.

\begin{tabular}{|c|c|c|c|c|c|c|}
\hline Study (Journal) & Study Type & Publication & Country & Study Groups & Study Period & Patient Selection \\
\hline $\begin{array}{l}\text { Kallen et al., } \\
2010 \text { [13] }\end{array}$ & $\begin{array}{l}\text { Retrospective } \\
\text { cohort }\end{array}$ & $\begin{array}{l}\text { Peer-reviewed } \\
\text { article }\end{array}$ & USA & $\mathrm{N} / \mathrm{A}$ & July 2005-February 2008 & $\begin{array}{l}\text { All patients who } \\
\text { underwent CTPA }\end{array}$ \\
\hline $\begin{array}{l}\text { Uehara et al., } \\
2011[16]\end{array}$ & $\begin{array}{l}\text { Retrospective } \\
\text { cohort }\end{array}$ & $\begin{array}{l}\text { Peer-reviewed } \\
\text { article }\end{array}$ & Japan & $\mathrm{N} / \mathrm{A}$ & $\begin{array}{l}\text { January 2005- } \\
\text { December } 2006\end{array}$ & $\begin{array}{l}\text { Consecutive patients with high } \\
\text { risk of PE based on symptoms } \\
\text { and clinical data (low PaO2, low } \\
\mathrm{PaCO} 2, \text { increased D-dimer, } \\
\text { ECG, ultrasonography) }\end{array}$ \\
\hline $\begin{array}{l}\text { Shahir, K et al., } \\
2013 \text { [15] }\end{array}$ & Case-control & $\begin{array}{l}\text { Peer-reviewed } \\
\text { article }\end{array}$ & USA & $\begin{array}{l}\text { Study: PE-positive } \\
\text { CTPA Control: } \\
\text { Randomly selected } \\
\text { negative CTPA }\end{array}$ & 2006-2008 & $\begin{array}{l}\text { Patients between } 18-40 \text { years old } \\
\text { who underwent CTPA (first scan } \\
\text { only if multiple). PE-positive } \\
\text { scans selected first, then randomly } \\
\text { selected controls (normal exam, } \\
\text { non-PE related findings) chosen } \\
\text { from same selection population. }\end{array}$ \\
\hline $\begin{array}{l}\text { Michalakis et al., } \\
2014 \text { [18] }\end{array}$ & $\begin{array}{l}\text { Prospective } \\
\text { cohort }\end{array}$ & $\begin{array}{l}\text { Peer-reviewed } \\
\text { article }\end{array}$ & Belgium & $\mathrm{N} / \mathrm{A}$ & $\begin{array}{l}\text { September 2010- } \\
\text { July } 2012\end{array}$ & $\begin{array}{l}\text { Consecutive patients who } \\
\text { underwent CTPA based on } \\
\text { clinical suspicion of PE }\end{array}$ \\
\hline $\begin{array}{l}\text { Shahir et al., } \\
2015 \text { [12] }\end{array}$ & $\begin{array}{l}\text { Retrospective } \\
\text { cohort }\end{array}$ & $\begin{array}{l}\text { Peer-reviewed } \\
\text { article }\end{array}$ & USA & $\mathrm{N} / \mathrm{A}$ & 2004-2012 & $\begin{array}{l}\text { All pregnant women who } \\
\text { underwent CTPA }\end{array}$ \\
\hline $\begin{array}{l}\text { Atalay et al., } \\
2011[11] \\
\text { (Clin Rad) }\end{array}$ & $\begin{array}{l}\text { Retrospective } \\
\text { cohort }\end{array}$ & $\begin{array}{l}\text { Peer-reviewed } \\
\text { article }\end{array}$ & USA & $\mathrm{N} / \mathrm{A}$ & $\begin{array}{l}\text { January 2005- } \\
\text { March } 2006\end{array}$ & $\begin{array}{l}\text { Consecutive patients positive for } \\
\text { acute PE }\end{array}$ \\
\hline $\begin{array}{l}\text { Atalay et al., } 2011 \\
\text { [14] } \\
\text { (J Cardiol } \\
\text { Comput Tomogr) }\end{array}$ & $\begin{array}{l}\text { Retrospective } \\
\text { cohort }\end{array}$ & $\begin{array}{l}\text { Peer-reviewed } \\
\text { article }\end{array}$ & USA & $\mathrm{N} / \mathrm{A}$ & $\begin{array}{l}\text { February 2010- } \\
\text { March } 2010\end{array}$ & $\begin{array}{l}\text { Patients who presented with chest } \\
\text { pain, hypoxemia, tachycardia, } \\
\text { shortness of breath or variations } \\
\text { of these as indication for } \\
\text { their CTPA }\end{array}$ \\
\hline $\begin{array}{l}\text { Hendriks et al., } \\
2019[20]\end{array}$ & $\begin{array}{l}\text { Retrospective } \\
\text { cohort }\end{array}$ & $\begin{array}{l}\text { Peer-reviewed } \\
\text { article }\end{array}$ & Netherlands & $\mathrm{N} / \mathrm{A}$ & Not specified & $\begin{array}{l}\text { Consecutive non-pregnant female } \\
\text { patients who underwent CTPA }\end{array}$ \\
\hline $\begin{array}{l}\text { Patel et al., } \\
2007 \text { [23] }\end{array}$ & $\begin{array}{l}\text { Retrospective } \\
\text { cohort }\end{array}$ & $\begin{array}{l}\text { Conference } \\
\text { abstract }\end{array}$ & $\begin{array}{c}\text { Not } \\
\text { specified }\end{array}$ & $\mathrm{N} / \mathrm{A}$ & Not specified & Not specified \\
\hline $\begin{array}{l}\text { Cowell \& } \\
\text { Sheridan, } \\
2012[22]\end{array}$ & $\begin{array}{l}\text { Retrospective } \\
\text { cohort }\end{array}$ & $\begin{array}{c}\text { Conference } \\
\text { abstract }\end{array}$ & $\begin{array}{c}\text { Not } \\
\text { specified }\end{array}$ & $\mathrm{N} / \mathrm{A}$ & January 2012-April 2012 & Patients who underwent CTPA \\
\hline $\begin{array}{l}\text { Atweh et al., } \\
2012[21]\end{array}$ & $\begin{array}{l}\text { Retrospective } \\
\text { cohort }\end{array}$ & $\begin{array}{l}\text { Conference } \\
\text { abstract }\end{array}$ & $\begin{array}{c}\text { Not } \\
\text { specified }\end{array}$ & $\mathrm{N} / \mathrm{A}$ & 2005-2011 & $\begin{array}{c}\text { All pediatric patients ( } 0-25 \text { years) } \\
\text { with a PE-positive CTPA }\end{array}$ \\
\hline $\begin{array}{l}\text { Ho et al., } \\
2019 \text { [17] }\end{array}$ & $\begin{array}{l}\text { Retrospective } \\
\text { and prospective } \\
\text { cohort }\end{array}$ & $\begin{array}{c}\text { Conference } \\
\text { abstract }\end{array}$ & England & $\begin{array}{c}\text { A: Patients who } \\
\text { underwent CTPA } \\
\text { (retrospective; } \\
n=153 \text { ) } \\
\text { B: Patients assessed } \\
\text { for dose for } \\
\text { standard and } \\
\text { reduced scan } \\
\text { coverage } \\
\text { (prospective; } \mathrm{n}=23 \text { ) }\end{array}$ & $\begin{array}{l}\text { A: Nov } 2018 \\
\text { B: December } 2018- \\
\text { January } 2019\end{array}$ & Patients who underwent CTPA \\
\hline $\begin{array}{l}\text { Chen et al., } \\
2019 \text { [19] }\end{array}$ & $\begin{array}{l}\text { Retrospective } \\
\text { cohort }\end{array}$ & $\begin{array}{l}\text { Conference } \\
\text { abstract }\end{array}$ & Australia & $\mathrm{N} / \mathrm{A}$ & Not specified & $\begin{array}{l}\text { Consecutive patients with a } \\
\text { PE-positive CTPA }\end{array}$ \\
\hline
\end{tabular}

$\mathrm{N} / \mathrm{A}=$ not applicable; $\mathrm{PE}$ = pulmonary embolism; CTPA = computed tomography pulmonary angiography. 
Table 2. CTPA type, z-axis used and associated reduction in mean scan length and radiation dose in included studies.

\begin{tabular}{|c|c|c|c|c|c|}
\hline $\begin{array}{c}\text { Study } \\
\text { (Journal) }\end{array}$ & CT Scan Type & $\begin{array}{l}\text { Reduced Scan } \\
\text { Window Used }\end{array}$ & $\begin{array}{c}\text { Was the Optimal } \\
\text { Scan Range } \\
\text { Evaluated to } \\
\text { Capture All PE } \\
\text { Diagnoses/Filling } \\
\text { Defects? }\end{array}$ & $\begin{array}{c}\text { Reduction in } \\
\text { Mean Scan Length } \\
\text { (cm, \% Reduction) }\end{array}$ & $\begin{array}{c}\text { Reduction in } \\
\text { Radiation Dose (\%) }\end{array}$ \\
\hline $\begin{array}{l}\text { Kallen et al., } \\
2010[13]\end{array}$ & 64-row MDCT & $\begin{array}{l}\text { Above the aortic arch to } \\
\text { below inferior-most aspect } \\
\text { of the heart }\end{array}$ & $\mathrm{N}$ & $9.6(37 \%)$ & $\mathrm{N} / \mathrm{A}$ \\
\hline $\begin{array}{l}\text { Uehara et al., } \\
2011 \text { [16] }\end{array}$ & 16-slice MDCT & $\begin{array}{c}\text { Top of aortic arch to below } \\
\text { the under surface of } \\
\text { the heart }\end{array}$ & $\mathrm{N}$ & $21.90 \%$ & 22 \\
\hline $\begin{array}{l}\text { Shahir et al., } \\
2013 \text { [15] }\end{array}$ & $\begin{array}{l}\text { 16-row and 64-row } \\
\text { MDCT }\end{array}$ & $\begin{array}{c}\text { Top of aortic arch to below } \\
\text { the level of the heart }\end{array}$ & $\mathrm{N}$ & $\begin{array}{c}11(42 \%) \text {; calculated } \\
\text { based on } 15 \text { different } \\
\text { consecutive patients } \\
\text { who underwent } \\
\text { CTPA }\end{array}$ & $\begin{array}{l}\text { 60; based on different } \\
15 \text { consecutive } \\
\text { patients who } \\
\text { underwent CTPA) }\end{array}$ \\
\hline $\begin{array}{l}\text { Michalakis } \\
\text { et al., } 2014 \text { [18] }\end{array}$ & $\begin{array}{l}\text { 16-section and } \\
\text { 64-row MDCT }\end{array}$ & $\begin{array}{l}10 \mathrm{~cm} \text { scan length starting } \\
\text { from the bottom of } \\
\text { aortic arch }\end{array}$ & $\mathrm{N}$ & $19.6(52 \%)$ & 69 \\
\hline $\begin{array}{l}\text { Shahir et al., } \\
2015 \text { [12] }\end{array}$ & $\begin{array}{l}\text { 16-row and 64-row } \\
\text { MDCT }\end{array}$ & $\begin{array}{c}\text { Top of aortic arch to below } \\
\text { the level of the heart }\end{array}$ & $\mathrm{N}$ & $15(42 \%)$ & $\begin{array}{c}71 ; \text { calculated based } \\
\text { on } 36 \text { consecutive } \\
\text { non-pregnant adult } \\
\text { patients who } \\
\text { underwent CTPA }\end{array}$ \\
\hline $\begin{array}{l}\text { Atalay et al., } \\
2011[11] \\
\text { (Clin Rad) }\end{array}$ & 16-row MDCT & $\begin{array}{c}\text { A: } 14.2 \mathrm{~cm} \text { scan length } \\
\text { centered } 4.1 \mathrm{~cm} \text { below the } \\
\text { carina (capture at least } \\
\text { one PE) } \\
\text { B: Top of aorta to bottom of } \\
\text { the heart }\end{array}$ & $\begin{array}{l}\text { A: } Y \\
\text { B: } N\end{array}$ & $\begin{array}{l}\text { A: } 11.7(44 \%) \\
\text { B: } 9.9(38 \%)\end{array}$ & $\mathrm{N} / \mathrm{A}$ \\
\hline $\begin{array}{l}\text { Atalay et al., } \\
2011 \text { [14] } \\
\text { (J Cardiol } \\
\text { Comput } \\
\text { Tomogr) }\end{array}$ & $\begin{array}{l}\text { 16-row and } \\
\text { 64-row MDCT }\end{array}$ & $\begin{array}{l}14.2 \mathrm{~cm} \text { scan length } \\
\text { centered } 4.1 \mathrm{~cm} \text { below the } \\
\text { carina (based on previous } \\
\text { study which optimized } \\
\text { scan length to capture } \\
\text { all PE) }\end{array}$ & $\mathrm{N}$ & $13.8(49 \%)$ & $\mathrm{N} / \mathrm{A}$ \\
\hline $\begin{array}{l}\text { Hendriks et al., } \\
2019 \text { [20] }\end{array}$ & $\begin{array}{c}\text { A: 64-slice MDCT } \\
\text { B: } 2 \times 128 \text {-slice DSCT } \\
\text { C: } 2 \times 128 \text {-slice DSCT } \\
\text { D: } 2 \times 192 \text {-slice DSCT }\end{array}$ & $\begin{array}{l}\text { A-B: Lung apex to the top } \\
\text { of the most } \\
\text { caudal diaphragm }\end{array}$ & A-B: N & $\begin{array}{l}\text { A: } 33 \% \\
\text { B: } 30 \% \\
\text { C: } 30 \% \\
\text { D: } 31 \%\end{array}$ & $\begin{array}{l}\text { A: } 26 \% \\
\text { B: } 25 \% \\
\text { C: } 26 \% \\
\text { D: } 23 \%\end{array}$ \\
\hline $\begin{array}{l}\text { Patel et al., } \\
2007[23]\end{array}$ & Not specified & $\begin{array}{l}\text { Top of the aortic arch to } \\
\text { below the heart }\end{array}$ & $\mathrm{N}$ & $\mathrm{N} / \mathrm{A}$ & 48 \\
\hline $\begin{array}{c}\text { Cowell \& } \\
\text { Sheridan, } 2012 \\
\text { [22] }\end{array}$ & Not specified & $\begin{array}{c}\text { Superior aspect of the aortic } \\
\text { arch to the inferior aspect of } \\
\text { the heart }\end{array}$ & $\mathrm{N}$ & Not specified & Not specified \\
\hline $\begin{array}{l}\text { Atweh et al., } \\
2012 \text { [21] }\end{array}$ & Not specified & $\begin{array}{c}\text { A: Patients without } \\
\text { congenital heart disease: } \\
14 \mathrm{~cm} \text { scan length centered } \\
3.5 \mathrm{~cm} \text { below the carina } \\
\text { (captures } 100 \% \text { of all } \\
\text { filling defects) } \\
\text { B: Patients with CHD: } 8 \mathrm{~cm} \\
\text { scan length centered } 5 \mathrm{~cm} \\
\text { below the carina (captures at } \\
\text { least } 1 \text { filling defect) }\end{array}$ & $\begin{array}{l}\text { A: } Y \\
\text { B: } Y\end{array}$ & $\begin{array}{l}\text { A: } 20 \% \\
\text { B: } 40 \%\end{array}$ & Not specified \\
\hline $\begin{array}{l}\text { Ho et al., } \\
2019[17]\end{array}$ & Not specified & $\begin{array}{c}\text { Humeral heads to lung } \\
\text { bases (excludes lung apices) }\end{array}$ & $\mathrm{N}$ & $\begin{array}{c}\text { A \& B: } 14.9 \mathrm{~cm} \\
(49.6 \%)\end{array}$ & $\begin{array}{l}\text { A: N/A } \\
\text { B: } 21\end{array}$ \\
\hline $\begin{array}{l}\text { Chen et al., } \\
2019 \text { [19] }\end{array}$ & Not specified & $\begin{array}{l}14.7 \mathrm{~cm} \text { length starting } \\
\text { superiorly at the top of the } \\
\text { aortic arch (no } \\
\text { rationale provided) }\end{array}$ & $\mathrm{N}$ & $\mathrm{N} / \mathrm{A}$ & $\mathrm{N} / \mathrm{A}$ \\
\hline
\end{tabular}


Six different reduced CTPA z-axes were pre-defined and assessed in the included studies, as depicted in Figure 2. Seven studies used reduced $z$-axis coverage from the top of the aortic arch extending to below the heart (window A in Figure 2) [11-13,15,16,22,23]. One study analyzed a $10 \mathrm{~cm} z$-axis length beginning superiorly from the bottom of the aortic arch (window B in Figure 2) [18]. One study used a $14.2 \mathrm{~cm} z$-axis length centered $4.1 \mathrm{~cm}$ below the carina (window $C$ in Figure 2) [14]. One study used a reduced scan window from the humeral heads to the lung bases, excluding the lung apices (window D in Figure 2) [17]. One study used a $14.7 \mathrm{~cm}$ scan length starting at the top of the aortic arch (window E in Figure 2) [19]. Finally, one study used a scan window from the lung apices to the most caudal diaphragm (window F in Figure 2) [20].

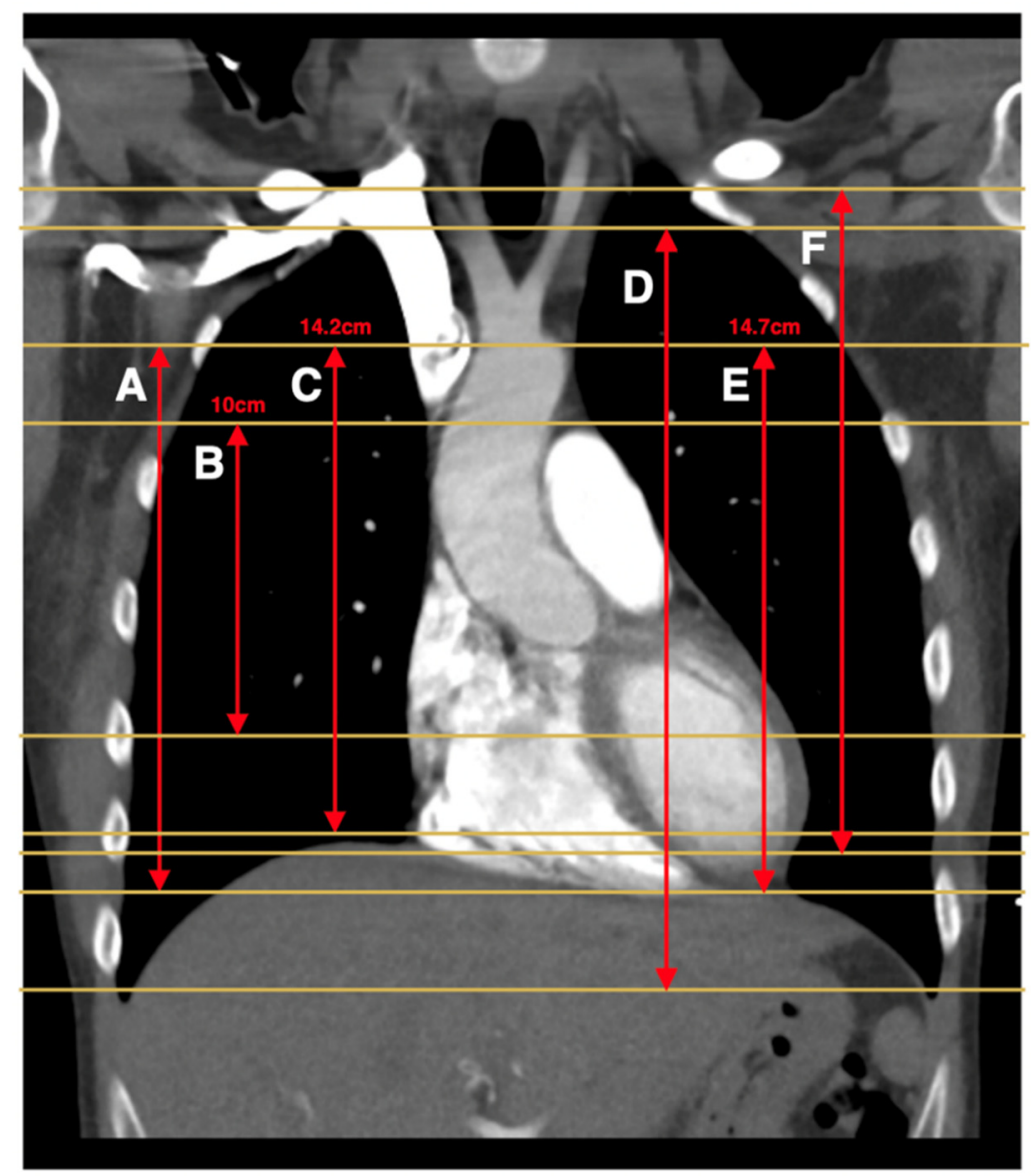

Figure 2. CTPA $z$-axis coverages assessed by studies: (A) top of the aortic arch to below the heart; (B) $10 \mathrm{~cm}$ window starting from the bottom of the aortic arch; (C) $14.2 \mathrm{~cm}$ window centered $4.1 \mathrm{~cm}$ below the carina; (D) humeral heads to lung bases (excluding apices); (E) $14.7 \mathrm{~cm}$ window starting from the top of the aortic arch; (F) top of the lung apices to most caudal diaphragm.

Additionally, two studies retrospectively determined the minimum optimal scan window to capture all PE diagnoses and/or filling defects, based on a dataset of CTPA scans which were positive for PE [11,21]. One study assessed 100 consecutive CTPA scans positive for $\mathrm{PE}$ and found that a $14.2 \mathrm{~cm} z$-axis length centered $4.1 \mathrm{~cm}$ below the carina captured at least one filling defect (i.e., all PE diagnoses), and an $18 \mathrm{~cm}$ scan length centered $4 \mathrm{~cm}$ below the carina captured all filling defects [11]. The second study included 45 CTPA scans positive for PE in patients aged 0-25 years and found that an $8 \mathrm{~cm} z$-axis length centered $5 \mathrm{~cm}$ below the carina captured at least one filling defect, and a $14 \mathrm{~cm} z$-axis length centered $3.5 \mathrm{~cm}$ below the carina captured all filling defects [21].

Of the 12 studies that reported the yield of PE diagnoses and/or filling defects with a pre-defined reduced scan window [11-20,22,23], two studies each reported one case of 
missed PE diagnosis (based on at least one filling defect) [18,19] (Table 3). Specifically, in one study, one diagnosis of a sub-segmental PE out of $57 \mathrm{PE}$ diagnoses (1.8\%) was missed when the $z$-axis was reduced to a $10 \mathrm{~cm}$ window starting from the bottom of the aortic arch [18], and in another study, one sub-segmental PE out of 200 PE diagnoses $(0.5 \%)$ was missed when a $14.7 \mathrm{~cm} z$-axis starting from the top of the aortic arch was used [19]. No PE diagnoses were missed when the $z$-axis was reduced from the top of the aortic arch to the below of the heart [11-13,15,16,20,22,23], from the humeral heads to the lung bases [17] or when a $14.2 \mathrm{~cm} z$-axis was centered $4.1 \mathrm{~cm}$ below the carina [14].

Table 3. Study outcomes: yield of PE diagnoses, filling defects and other (non-PE) diagnoses with reduced CTPA scan coverage.

\begin{tabular}{|c|c|c|c|c|c|c|c|c|c|}
\hline $\begin{array}{c}\text { Study } \\
\text { (Journal) }\end{array}$ & $\begin{array}{c}\text { Total } \\
\text { Number } \\
\text { of CTPAs } \\
\text { Analysed }\end{array}$ & $\begin{array}{l}\text { Number } \\
\text { of CTPAs } \\
\text { Excluded }\end{array}$ & Reasons for Exclusion & $\begin{array}{l}\text { Number } \\
\text { of CTPAs } \\
\text { Included }\end{array}$ & $\begin{array}{c}\text { PE-Positive } \\
\text { CTPAs (\% } \\
\text { of Included } \\
\text { Scans) }\end{array}$ & $\begin{array}{l}\text { Number of } \\
\text { PE } \\
\text { Diagnoses } \\
\text { Missed with } \\
\text { Reduced } \\
\text { Scan } \\
\text { Window (\% } \\
\text { of All PEs) }\end{array}$ & $\begin{array}{l}\text { Number } \\
\text { of Filling } \\
\text { Defects } \\
\text { Missed }\end{array}$ & $\begin{array}{l}\text { Total } \\
\text { Number } \\
\text { of Other } \\
\text { Findings }\end{array}$ & $\begin{array}{l}\text { Number of } \\
\text { Other } \\
\text { Findings } \\
\text { Missed with } \\
\text { Reduced } \\
\text { Scan } \\
\text { Window (\% } \\
\text { of All Other } \\
\text { Findings) }\end{array}$ \\
\hline $\begin{array}{c}\text { Kallen et al. } \\
2010 \text { [13] }\end{array}$ & 1734 & 0 & Not applicable & 1734 & $295(17)$ & 0 & $\begin{array}{c}\text { Not } \\
\text { specified }\end{array}$ & $\begin{array}{c}\text { Not } \\
\text { specified }\end{array}$ & $\begin{array}{c}\text { Not } \\
\text { specified }\end{array}$ \\
\hline $\begin{array}{c}\text { Uehara et al., } \\
2011 \text { [16] }\end{array}$ & 75 & 0 & Not applicable & 75 & $75(100)$ & 0 & $2(2.6 \%)$ & $\begin{array}{c}\text { Not } \\
\text { specified }\end{array}$ & $\begin{array}{c}\text { Not } \\
\text { specified }\end{array}$ \\
\hline $\begin{array}{l}\text { Shahir et al., } \\
2013 \text { [15] }\end{array}$ & 878 & $678(77 \%)$ & $\begin{array}{l}\text { Suboptimal opacification } \\
\text { of pulmonary arteries, } \\
\text { compromised evaluation } \\
\text { due to breathing artefact } \\
\text { (n=112); negative for PE; } \\
\text { not randomly selected } \\
\text { for control group }\end{array}$ & 200 & $86(43.7)$ & 0 & $\begin{array}{c}\text { Not } \\
\text { specified }\end{array}$ & $\begin{array}{l}392(1.96 \\
\text { findings } \\
\text { per scan) }\end{array}$ & $7(1.8)$ \\
\hline $\begin{array}{l}\text { Michalakis } \\
\text { et al., 2014 } \\
{[18]}\end{array}$ & 253 & $6(0.023 \%)$ & $\begin{array}{l}\text { Poor arterial } \\
\text { enhancement }\end{array}$ & 247 & $57(23.4)$ & $\begin{array}{l}1 \text { (1.8; sub- } \\
\text { segmental) }\end{array}$ & $\begin{array}{c}\text { Not } \\
\text { specified }\end{array}$ & $\begin{array}{l}343(1.39 \\
\text { findings } \\
\text { per scan) }\end{array}$ & $48(14)$ \\
\hline $\begin{array}{l}\text { Shahir et al., } \\
2015 \text { [12] }\end{array}$ & 95 & $11(8.64 \%)$ & $\begin{array}{c}\text { Suboptimal contrast } \\
\text { opacification; respiratory } \\
\text { motion artefacts }\end{array}$ & 84 & $\begin{array}{l}2(2.3 ; \\
\text { segmental } \\
[\mathrm{n}=2])\end{array}$ & 0 & $\begin{array}{c}\text { Not } \\
\text { specified }\end{array}$ & $\begin{array}{c}76(0.9 \\
\text { findings } \\
\text { per scan })\end{array}$ & $4(5.26)$ \\
\hline $\begin{array}{l}\text { Atalay et al., } \\
2011[11] \\
\text { (Clin Rad) }\end{array}$ & 95 & $11(8.64 \%)$ & $\begin{array}{c}\text { Suboptimal contrast } \\
\text { opacification; respiratory } \\
\text { motion artefacts }\end{array}$ & 84 & $\begin{array}{c}2(2.3 ; \\
\text { segmental } \\
[n=2])\end{array}$ & 0 & $\begin{array}{c}\text { Not } \\
\text { specified }\end{array}$ & $\begin{array}{c}76(0.9 \\
\text { findings } \\
\text { per scan })\end{array}$ & $4(5.26)$ \\
\hline $\begin{array}{c}\text { Atalay et al., } \\
2011 \text { [14] } \\
\text { (J Cardiol } \\
\text { Comput } \\
\text { Tomogr) }\end{array}$ & 95 & $11(8.64 \%)$ & $\begin{array}{c}\text { Suboptimal contrast } \\
\text { opacification; respiratory } \\
\text { motion artefacts }\end{array}$ & 84 & $\begin{array}{l}2(2.3 ; \\
\text { segmental } \\
[n=2])\end{array}$ & 0 & $\begin{array}{c}\text { Not } \\
\text { specified }\end{array}$ & $\begin{array}{c}76(0.9 \\
\text { findings } \\
\text { per scan })\end{array}$ & $4(5.26)$ \\
\hline $\begin{array}{l}\text { Hendriks } \\
\text { et al., } 2019 \\
{[20]}\end{array}$ & 95 & $11(8.64 \%)$ & $\begin{array}{c}\text { Suboptimal contrast } \\
\text { opacification; respiratory } \\
\text { motion artefacts }\end{array}$ & 84 & $\begin{array}{c}2(2.3 ; \\
\text { segmental } \\
[\mathrm{n}=2])\end{array}$ & 0 & $\begin{array}{c}\text { Not } \\
\text { specified }\end{array}$ & $\begin{array}{c}76(0.9 \\
\text { findings } \\
\text { per scan })\end{array}$ & $4(5.26)$ \\
\hline $\begin{array}{l}\text { Patel et al., } \\
2007 \text { [23] }\end{array}$ & 95 & $11(8.64 \%)$ & $\begin{array}{c}\text { Suboptimal contrast } \\
\text { opacification; respiratory } \\
\text { motion artefacts }\end{array}$ & 84 & $\begin{array}{c}2(2.3 \\
\text { segmental } \\
[\mathrm{n}=2])\end{array}$ & 0 & $\begin{array}{c}\text { Not } \\
\text { specified }\end{array}$ & $\begin{array}{c}76(0.9 \\
\text { findings } \\
\text { per scan })\end{array}$ & $4(5.26)$ \\
\hline $\begin{array}{l}\text { Cowell \& } \\
\text { Sheridan, } \\
2012[22]\end{array}$ & 200 & $\begin{array}{c}161 \\
(80.5 \%)\end{array}$ & Negative for PE & 39 & $39(100)$ & 0 & $\begin{array}{c}\text { Not } \\
\text { specified }\end{array}$ & $\begin{array}{c}\text { Not } \\
\text { specified }\end{array}$ & $\begin{array}{l}3 \text { patients } \\
\text { with other } \\
\text { findings } \\
\text { (number of } \\
\text { missed } \\
\text { findings not } \\
\text { specified) }\end{array}$ \\
\hline $\begin{array}{l}\text { Atweh et al., } \\
2012 \text { [21] }\end{array}$ & $\begin{array}{c}\text { Not } \\
\text { specified }\end{array}$ & $\begin{array}{c}\text { Not } \\
\text { specified }\end{array}$ & Negative for PE & 45 & $45(100)$ & $\begin{array}{l}\text { A: } 0 \\
\text { B: } 0\end{array}$ & $\begin{array}{c}\text { A: } 0 \\
\text { B: Not } \\
\text { specified }\end{array}$ & $\begin{array}{c}\text { Not } \\
\text { specified }\end{array}$ & $\begin{array}{c}\text { Not } \\
\text { specified }\end{array}$ \\
\hline $\begin{array}{l}\text { Ho et al., } \\
2019 \text { [17] }\end{array}$ & $\begin{array}{l}\text { A: } 153 \\
\text { B: } 23\end{array}$ & $\begin{array}{c}\text { Not } \\
\text { specified }\end{array}$ & Not specified & $\begin{array}{l}\text { A: } 153 \\
\text { B: } 23\end{array}$ & $\begin{array}{l}\text { A: } 29(19) \\
\text { B: Not } \\
\text { specified }\end{array}$ & 0 & $\begin{array}{c}\text { Not } \\
\text { specified }\end{array}$ & $\begin{array}{c}\text { Not } \\
\text { specified }\end{array}$ & $\begin{array}{c}\text { Not } \\
\text { specified }\end{array}$ \\
\hline $\begin{array}{c}\text { Chen et al., } \\
2019 \text { [19] }\end{array}$ & 200 & $\begin{array}{c}\text { Not } \\
\text { specified }\end{array}$ & Not specified & 200 & $200(100)$ & $\begin{array}{l}1(0.5 ; \text { sub- } \\
\text { segmental) }\end{array}$ & $2(1 \%)$ & $\begin{array}{c}\text { Not } \\
\text { specified }\end{array}$ & $\begin{array}{c}\text { Not } \\
\text { specified }\end{array}$ \\
\hline
\end{tabular}


Of the three studies $[11,16,19]$ that reported on the diagnostic yield of their reduced scan windows in capturing all filling defects, two studies reported instances of missed filling defects. In one study, $2.7 \%$ of filling defects $(n=2)$ were missed in two patients when the $z$-axis was reduced to a $z$-axis from the top of the aortic arch to under-the-surface of the heart; however, the diagnosis of PE was not missed due the presence of other filling defects within the scan field [16]. In the second study, $1 \%$ of filling defects $(n=2)$ were missed in two patients using a fixed $14.7 \mathrm{~cm}$ z-axis starting from the top of the aortic arch [19]. Of the two filling defects missed, one was located outside the reduced scan window and resulted in a missed diagnosis of PE [19].

Six studies reported on the yield of diagnoses other than PE; all found that other diagnoses were missed when the scan window was reduced (Table 3) [12,14,15,18,20,22]. When the scan window covered the area from the top of the aortic arch to below the heart, the number of missed findings was as follows: 7 out of 392 other findings missed $(1.8 \%)$ [15], 4 out of 76 other findings missed (5.3\%) [12], and one study reported that other findings were missed in three patients without specifying the number of missed diagnoses [22]. When the scan window reached from the lung apices to the most caudal diaphragm, four other findings were missed [20]. When the scan window was limited to a $10 \mathrm{~cm}$ scan length beginning at the bottom of the aortic arch, 48 of 343 (14\%) other findings were missed [18]. When the scan window was limited to a $14.2 \mathrm{~cm}$ scan length centered $4.1 \mathrm{~cm}$ below the carina, 63 of 604 (10.4\%) other diagnoses were missed [14]. The most common other diagnoses missed were thyroid nodules $(n=27)$, hiatal hernia $(n=21)$ and cholelithiasis $(n=12)$, which were likely not associated with the patients' symptoms at presentation.

Most of the included studies had a high risk of bias (Table S3). Using our modified risk of bias tool, three studies scored 6 or more points and were categorized as having a low risk of bias $[12,15,18]$, and ten studies scored less than 6 points and were categorized as having a high risk of bias [11,13,14,16,17,19-23].

\section{Discussion}

This is the first systematic review of the utility of reduced CTPA z-axis for the diagnosis of PE. Of the different types of $z$-axis length assessed, from the top of the aortic arch to below the heart was the most frequently investigated by seven studies, and no PE diagnoses were missed with this z-axis length. Diagnosis of a sub-segmental PE was missed once with two different reduced $z$-axis windows-a $10 \mathrm{~cm}$ window starting from the bottom of the arch and a $14.7 \mathrm{~cm}$ window starting from the top of the aortic arch. Although two studies reported missing filling defects, in one of these studies, despite missing two (2.7\%) filling defects, no PE diagnoses were missed due to the presence of additional diagnostic filling defects within the reduced scan window [16]. In all studies that reported on the yield of other diagnoses, other diagnoses were missed regardless of the type of reduced $z$-axis coverage.

Both PE diagnoses that were missed when using different z-axes with an absolute length were isolated sub-segmental PE. The benefit of treating isolated sub-segmental PEs is unclear [24]. The guidelines of the American College of Chest Physicians, for example, suggest that it might be reasonable not to anticoagulated a patient with an isolated subsegmental PE if there is no evidence of proximal lower extremity deep vein thrombosis or evidence of thrombus elsewhere (e.g., upper extremity clot) and the risk of recurrence is considered low [25].

The major benefit derived from reducing the anatomical scan window is the reduced radiation compared to a full-length scan. While estimating the exact radiation dose reduction is complex due to variability based on different tissue densities, the effective radiation dose is effectively directly proportional to scan length [14]. Since areas such as the lung are impacted less by radiation than tissue-dense areas such as the abdomen [14], reducing the scan window to exclude such areas, which is achieved by all scan windows assessed in our systematic review, greatly reduces radiation exposure. While this is beneficial for all 
patients, young and pregnant women stand to benefit the most. Bismuth breast shields can reduce radiation dosage by 26-41\% [5], but have varied use internationally-within the United States of America, for example, these shields are not routinely used in practice [26]. For a fetus, the radiation dosage associated with a mother's CTPA is significantly less than the levels required to produce teratogenic effects [27]; however, little is known about the long-term risk and potential genetic damage induced [28]. While in general, the benefits of CTPA seem to outweigh the associated risks in patients with a high pre-test probability of PE, it is desirable to minimize radiation exposure associated with CTPAs, especially in pregnant women, for example by using a reduced anatomical scan window.

Some incidental findings were missed with limited CTPA scan coverages. While some CTPA findings other than PE may offer an alternative diagnosis for the patient's presentation (e.g., pleural effusion), incidental findings on CTPAs (e.g., of a pulmonary or thyroid nodule) can also lead to over-investigation and over-treatment, which potentially exposes the patient to unnecessary risks [29]. A reduced detection rate of incidental findings on CTPA is therefore not a reason not to use a reduced anatomical cover scan.

This systematic review included two studies [11,21] that did not use predefined anatomical landmarks to reduce the scan window but evaluated the optimal scan range measured in centimeters to capture all identified PEs and/or filling defects. The optimized scan windows that captured at least one filling defect were a $14.2 \mathrm{~cm}$ scan length centered $4.1 \mathrm{~cm}$ below the carina (based on adult patients) [11] and an $8 \mathrm{~cm}$ scan length centered $5 \mathrm{~cm}$ below the carina (based on pediatric patients) [21]. The former scan window was subsequently validated in a second study (also included in this systematic review), which found that no PE diagnoses were missed when this scan window was used [14]. Scan windows based on absolute measures and not easily identifiable anatomical landmarks are potentially problematic in clinical practice. Firstly, radiographers routinely use anatomical landmarks to determine the CTPA window [30]. Secondly, absolute measures may not be applicable to all population groups, as patients may have significant variations in anatomy and thorax length. These variations may be due to patient sex, size and underlying medical conditions (such as chronic obstructive pulmonary disease). A z-axis defined by anatomical landmarks at both ends is generally applicable independently of the individual's thorax length. Furthermore, both studies that evaluated the optimal scan range measured in centimeters were performed at a single institution; one study included only adult ED patients [11] and one study included only pediatric patients [21]. Therefore, these results cannot be generalized to other populations.

This study has several limitations. Most of the studies had a high risk of bias. Of the included studies published in peer-reviewed journals, five of eight studies did not specify whether scans were excluded for poor image quality $[11,13,14,16,20]$. Only three studies specified that radiologists were blinded to the original CTPA report $[12,15,18]$, and one study did not indicate the years of experience of the reporting doctor [16].

Five of the twelve included studies were conference abstracts $[17,19,21-23]$. Conference abstracts are characterized by a dearth of detailed information due to the restricted format as well as lack of peer review when compared to studies published in peer-reviewed journals. None of the included abstracts provided any details pertaining to the items in our risk of bias assessment, and hence, all scored zero points (high risk of bias).

Most of the proposed z-axis coverages were only assessed in one study each with a relatively low sample size of CTPAs. However, the CTPA scan coverage from the top of the aortic arch to below the heart was assessed in seven studies and did not miss any PE diagnoses.

\section{Conclusions}

This systematic review demonstrates that there is potential to limit the scan window of a CTPA without reducing the diagnostic yield for PE. Physicians should consider utilizing the option to limit the scan coverage of a CTPA, particularly in population groups where radiation exposure is of special concern, such as pregnant women. The scan coverage from 
the top of the aortic arch to below the heart may be the best approach in achieving radiation dose reduction without impairing diagnostic yield.

Supplementary Materials: The following are available online at https://www.mdpi.com/article/10 .3390 / diagnostics11122179/s1, Supplementary Table S1: Search strategy performed in MEDLINE and EMBASE, Supplementary Table S2: Risk of bias assessment tool, Supplementary Table S3: Risk of bias assessment, Supplementary Table S4: List of excluded studies.

Author Contributions: Conceptualization, L.L. and C.C.D.; Methodology, C.C.D.; Investigation, A.Z. and R.N.; Writing—original draft preparation, A.Z.; Writing—review and editing, A.Z., R.N., L.L., A.K. and C.C.D.; Supervision, C.C.D. All authors have read and agreed to the published version of the manuscript.

Funding: This research received no specific grant from any funding agency in the public, commercial or not-for-profit sectors. C.C.D. was supported by a fellowship from the Australian National Health and Medical Research Council (NHMRC), grant number APP1123733. Open Access publishing of this article was supported by the Woolcock Institute of Medical Research.

Institutional Review Board Statement: Not applicable.

Informed Consent Statement: Not applicable.

Conflicts of Interest: The authors declare no conflict of interest.

\section{References}

1. Giordano, N.J.; Jansson, P.S.; Young, M.N.; Hagan, K.A.; Kabrhel, C. Epidemiology, pathophysiology, stratification, and natural history of pulmonary embolism. Tech. Vasc. Interv. Radiol. 2017, 20, 135-140. [CrossRef] [PubMed]

2. Estrada, Y.M.R.M.; Oldham, S.A. CTPA as the gold standard for the diagnosis of pulmonary embolism. Int. J. Comput. Assist. Radiol. Surg. 2011, 6, 557-563. [CrossRef]

3. Wiener, R.S.; Schwartz, L.M.; Woloshin, S. Time trends in pulmonary embolism in the United States: Evidence of overdiagnosis. Arch. Intern. Med. 2011, 171, 831-837. [CrossRef] [PubMed]

4. Osman, M.; Subedi, S.K.; Ahmed, A.; Khan, J.; Dawood, T.; Ríos-Bedoya, C.F.; Bachuwa, G. Computed tomography pulmonary angiography is overused to diagnose pulmonary embolism in the emergency department of academic community hospital. J. Community Hosp. Intern. Med. Perspect. 2018, 8, 6-10. [CrossRef] [PubMed]

5. Gillespie, C.; Foley, S.; Rowan, M.; Ewins, K.; NiAinle, F.; MacMahon, P. The OPTICA study (optimised computed tomography pulmonary angiography in pregnancy quality and safety study): Rationale and design of a prospective trial assessing the quality and safety of an optimised CTPA protocol in pregnancy. Thromb. Res. 2019, 177, 172-179. [CrossRef]

6. Kline, J.A.; Courtney, D.M.; Beam, D.M.; King, M.C.; Steuerwald, M. Incidence and predictors of repeated computed tomographic pulmonary angiography in emergency department patients. Ann. Emerg. Med. 2009, 54, 41-48. [CrossRef]

7. Champion, N.; Hogan, S.; Flemming, J. Assessing the prevalence of incidental findings identified by CTPA in women of reproductive age. Emerg. Med. Int. 2018, 2018, 1-5. [CrossRef]

8. Refaat, R.; El-Shinnawy, M.A. Does the anatomic distribution of acute pulmonary emboli at MDCT pulmonary angiography in oncology-population differ from that in non-oncology counterpart? Egypt. J. Radiol. Nucl. Med. 2013, 44, 463-474. [CrossRef]

9. Dobler, C.C. Overdiagnosis of pulmonary embolism: Definition, causes and implications. Breathe 2019, 15, 46-53. [CrossRef]

10. Page, M.J.; McKenzie, J.E.; Bossuyt, P.M.; Boutron, I.; Hoffmann, T.C.; Mulrow, C.D.; Shamseer, L.; Tetzlaff, J.M.; Akl, E.A.; Brennan, S.E.; et al. The PRISMA 2020 statement: An updated guideline for reporting systematic reviews. BMJ (Clin. Res. Ed.) 2021, 372, n71.

11. Atalay, M.K.; Walle, N.L.; Grand, D.J.; Mayo-Smith, W.W.; Cronan, J.J.; Egglin, T.K. Scan length optimization for pulmonary embolism at CT angiography: Analysis based on the three-dimensional spatial distribution of 370 emboli in 100 patients. Clin. Radiol. 2011, 66, 405-411. [CrossRef]

12. Shahir, K.; McCrea, J.M.; Lozano, L.A.S.; Goodman, L.R. Reduced z-axis technique for CT Pulmonary angiography in pregnancyvalidation for practical use and dose reduction. Emerg. Radiol. 2015, 22, 651-656. [CrossRef]

13. Kallen, J.A.; Coughlin, B.F.; O'Loughlin, M.T.; Stein, B. Reduced Z-axis coverage multidetector CT angiography for suspected acute pulmonary embolism could decrease dose and maintain diagnostic accuracy. Emerg. Radiol. 2010, 17, 31-35. [CrossRef]

14. Atalay, M.K.; Walle, N.L.; Egglin, T.K. Prevalence and nature of excluded findings at reduced scan length CT angiography for pulmonary embolism. J. Cardiovasc. Comput. Tomogr. 2011, 5, 325-332. [CrossRef]

15. Shahir, K.; Goodman, L.R.; Lam, C.A.; Midia, E.C. Dose reduction of $69 \%$ for computed tomography pulmonary angiography: Reduced $z$-axis computed tomography pulmonary angiography retains accuracy in those younger than 40 years. J. Comput. Assist. Tomogr. 2013, 37, 765-769. [CrossRef] [PubMed] 
16. Uehara, M.; Tanabe, N.; Funabashi, N.; Takaoka, H.; Ikari, J.; Toyama, S.; Shimizu, H.; Hoshino, S.; Sugiura, T.; Saito, M.; et al. Detailed distribution of acute pulmonary thromboemboli: Direct evidence for reduction of acquisition length and radiation dose for triple rule-out CT angiography. Int. J. Cardiol. 2011, 147, 234-238. [CrossRef]

17. Ho, A.; Mathew, S.; Harden, S. CTPAs: Reducing radiation dose without compromising diagnostic accuracy. Clin. Radiol. 2019, 74, e17. [CrossRef]

18. Michalakis, N.; Keyzer, C.; De Maertelaer, V.; Tack, D.; Gevenois, P.A. Reduced z-axis coverage in multidetector-row CT pulmonary angiography decreases radiation dose and diagnostic accuracy of alternative diseases. Br. Inst. Radiol. 2014, 87, 20130546. [CrossRef] [PubMed]

19. Chen, N.; Bhatti, J.; Rabinowitz, D. Tailoring CT pulmonary angiogram to different clinical scenarios. J. Med. Imaging Radiat. Oncol. 2019, 63, 53-54.

20. Hendriks, B.M.F.; Schnerr, R.S.; Milanese, G.; Jeukens, C.R.L.P.N.; Niesen, S.; Eijsvoogel, N.G.; Wildberger, J.E.; Das, M. Computed tomography pulmonary angiography during pregnancy: Radiation dose of commonly used protocols and the effect of scan length optimization. Korean J. Radiol. 2019, 20, 313-322. [CrossRef]

21. Atweh, L.; Orth, R.C.; Seghers, V.; Zhang, W.; Guillerman, R.P. Limited Z-axis coverage strategy for reducing radiation dose of CT pulmonary angiography for the diagnosis of pulmonary embolism in children. Pediatr. Radiol. 2012, 42 , S268.

22. Cowell, D.; Sheridan, S. Limited range CTPA-A safe way to reduce dose? Clin. Radiol. 2012, 67, S4. [CrossRef]

23. Patel, H.; Coughlin, B.F.; LaFrance, T.; O’Donnell-Moran, G. Comparison of Full Chest CTA with Limited CTA and Triple Rule-out CTA for PE Detection and Effective Dose Implications. In Proceedings of the Radiological Society of North America Annual Meeting, Chicago, IL, USA, 25-30 November 2007.

24. Carrier, M.; Righini, M.; Wells, P.S.; Perrier, A.; Anderson, D.R.; Rodger, M.A.; Pleasance, S.; Le Gal, G. Subsegmental pulmonary embolism diagnosed by computed tomography: Incidence and clinical implications. A systematic review and meta-analysis of the management outcome studies. J. Thromb. Haemost. 2010, 8, 1716-1722. [CrossRef] [PubMed]

25. Kearon, C.; Akl, E.A.; Ornelas, J.; Blaivas, A.; Jimenez, D.; Bounameaux, H.; Huisman, M.V.; King, C.S.; Morris, T.A.; Sood, N.; et al. Antithrombotic therapy for VTE disease: CHEST guideline and expert panel report. Chest 2016, 149, 315-352. [CrossRef]

26. Cogley, J.R.; Ghobrial, P.M.; Chandrasekaran, B.; Allen, S.B. Pulmonary embolism evaluation in the pregnant patient: A review of current imaging approaches. Semin. Ultrasound CT MRI 2012, 33, 11-17. [CrossRef] [PubMed]

27. Niemann, T.; Nicolas, G.; Roser, H.W.; Müller-Brand, J.; Bongartz, G. Imaging for suspected pulmonary embolism in pregnancywhat about the fetal dose? A comprehensive review of the literature. Insights Imaging 2010, 1, 361-372. [CrossRef] [PubMed]

28. Hancock, J.; Reed, W. A guide to foetal dose minimisation in computed tomography pulmonary angiography of the pregnant patient. Radiographer 2012, 59, 56-60. [CrossRef]

29. Hofmann, B. Diagnosing overdiagnosis: Conceptual challenges and suggested solutions. Eur. J. Epidemiol. 2014, 29, 599-604. [CrossRef]

30. Demircioğlu, A.; Stein, M.C.; Kim, M.-S.; Geske, H.; Quinsten, A.S.; Blex, S.; Umutlu, L.; Nassenstein, K. Detecting the pulmonary trunk in CT scout views using deep learning. Sci. Rep. 2021, 11, 10215. [CrossRef] [PubMed] 